\title{
Radiometric Imaging for Monitoring and Surveillance Issues
}

\author{
Francesco Soldovieri, ${ }^{1}$ Antonio Natale, ${ }^{1}$ Vladimir Gorishnyak, ${ }^{2}$ Andrey Pavluchenko, ${ }^{2}$ \\ Alexander Denisov, ${ }^{2}$ and Lijia Chen ${ }^{3}$ \\ ${ }^{1}$ Institute for Electromagnetic Sensing of the Environment, Consiglio Nazionale delle Ricerche (IREA-CNR), \\ Via Diocleziano 328, 80124 Napoli, Italy \\ ${ }^{2}$ State Research Center "Iceberg" (SRC), Avenue Lesya Kurbasa 2B, 03148 Kiev, Ukraine \\ ${ }^{3}$ School of Electronics and Information Engineering, Harbin Institute of Technology, P.O. Box 3054, Yikuang Street, \\ Nangang District, Harbin City, Heilongjiang Province, China \\ Correspondence should be addressed to Francesco Soldovieri; soldovieri.f@irea.cnr.it
}

Received 13 May 2013; Accepted 27 June 2013

Academic Editor: Rocco Pierri

Copyright (C) 2013 Francesco Soldovieri et al. This is an open access article distributed under the Creative Commons Attribution License, which permits unrestricted use, distribution, and reproduction in any medium, provided the original work is properly cited.

\begin{abstract}
This paper deals with the recent advances performed by State Research Center "Iceberg" (SRC) in the field of the passive imaging at millimeter wavelengths. In particular, first the paper describes the design and the realization of two systems working in $3 \mathrm{~mm}$ and $8 \mathrm{~mm}$ wave bands, respectively. Second, the measurements collected by the two systems are enhanced by means of simple data processing strategies developed by the Institute for Electromagnetic Sensing of the Environment (IREA-CNR).
\end{abstract}

\section{Introduction}

The capability of millimeter waves to propagate through fog, clouds, drizzle, dry snow, smoke, and other obstacles makes it possible to develop imaging systems able to tackle a wide arena of applicative problems, which cannot be solved by systems working at different bands as infrared and visible ones $[1,2]$. In the recent years, the main efforts have been focused on the necessities to achieve real- or near real time images of good quality in terms of spatial resolution and rejection of the clutter and noise.

The acquisition time is dependent on the number of sensors in the focal plane array and the speed of the scanning. In this frame, the better the sensitivity of the sensors is, the more it is possible to reduce the acquisition time; this is allowed by the integration time reduction by keeping at the same time a good image quality.

About the image quality, one of the crucial factors is the spatial resolution and, as well known, the diffraction limited spatial resolution, of a passive mm-wave imaging system is inversely proportional to the diameter (related to the probed wavelength) of the quasioptical antenna. Thus, the development of the practical $\mathrm{mm}$-wave imaging system aims at increasing the number of the high-sensitive receiving sensors in focal plane array and at using quasi-optical antenna with a diameter very large compared to that of the probed wavelength.

In this context, here we present two radiometric passive imaging systems designed and realized at SRC.

The first one is a 16-channel radiometric imaging system [3-5] working at $94 \mathrm{GHz}$ and able to obtain an image of the investigated scene in 10 seconds. The second one is a 32-channel $8 \mathrm{~mm}$ imaging system, working at frequency 33$38 \mathrm{GHz}$, which is able to obtain an image three times faster with better image quality compared to the first 16 channels system. After the description of the two systems, we will present some acquisition results and we will point out that the interpretability of the collected images can be improved by exploiting image processing strategies developed at IREACNR.

Therefore, the paper is organized as follows. Sections 2 and 3 are devoted to present the 16 channels system and 32 channels system, respectively. Section 4 presents several measurements collected by the two systems and how these images can be improved in terms of interpretability by simple processing strategies developed at IREA-CNR. Finally, conclusions follow. 


\section{The $3 \mathrm{~mm}$ Imaging System at $94 \mathrm{GHz}$}

The first prototype developed at SRC is a simple imaging system $[3,4]$ realized with a Cassegrain antenna with the driving mechanism, as shown in Figure 1. The radiometric sensor was a heterodyne receiver working in the frequency band 90$94 \mathrm{GHz}$. The half power beam width (HPBW) of the antenna is 1 degree and the scan step is 0.4 degree in both elevation and azimuth directions. Therefore, the radiometer is a step scan one; for the movement along one step about $200 \mathrm{~ms}$ are required while the integration time was set at $50 \mathrm{~ms}$, as result, the acquisition of one pixel measurement needs about $250 \mathrm{~ms}$.

The system works in the way described here. At the first position (let us say position 1), the radiometer measures a brightness temperature of the scene at the pixel, then it scans the azimuth direction by covering the angle between -45.8 and +45.8 degrees (228 pixels). After finishing the scanning of the azimuth line, the radiometer shifts one elevation step $(0.4$ degrees) and repeats the azimuth scan. Once the brightness image at the position 1 was taken, the radiometer was moved along the baseline by $2 \mathrm{~m}$ to the right (to position 2 ). When placing the radiometer at position 2 , the position and direction of the radiometer is checked thoroughly with a compass and inclinometer in order to minimize the pointing error. It was intended to reduce the complex camera calibration work by image processing. The image at position 2 was measured in the same scanning way as at position 1 . Therefore, the time amount of a horizontal scan is about $11 \mathrm{sec}$. The list of the technical parameters is given in Table 1 .

As a proof of the effectiveness of the system developed by SRC, we show here an image of the concealed object detection performed by the system (see Figure 2). In fact, passive detection of the concealed weapon and other objects under clothes is based on the fact that all bodies radiate, absorb, reflect, and propagate an electromagnetic energy on dependence of the features of the body as the material, shape, temperature, and condition of the surface, as well as on the frequency of the radiation. The clothes are more or less transparent to the millimeter waves, whereas the human body basically absorbs the millimeter wave radiation; differently, the material, of which the weapon is made, most completely reflects the surrounding radiation. As a result if the measurements are made indoor, the radiometric sensor is able to see the contrast between the radiation of a body with temperature around $309 \mathrm{~K}$ and the room temperature (indoor) around $297 \mathrm{~K}$. Thus, passive imaging at $3 \mathrm{~mm}$ wave band can represent an effective noninvasive remote-sensing method for the detection and recognition of weapon concealed under clothes. Of course, there will be other problems in case of realizing a real system for safety purposes, as described in [5].

\section{The $8 \mathrm{~mm}$ Passive Imaging System with 32 Sensors}

The second system developed by SRC [6-8] is an $8 \mathrm{~mm}$ imaging system equipped with an array of 32 sensors. The focal plane array is one of the main functional units of the imaging system and ensures the reception of the signals arriving from different directions of the surveyed scene. The array is built
TABLE 1: Instrument specifications for the experiment.

\begin{tabular}{lc}
\hline Center frequency & $94 \mathrm{GHz}$ \\
Band width & $1.7 \mathrm{GHz}$ \\
Polarization & Linear (vertical) \\
Integration time & Set to $50 \mathrm{~ms}$ \\
Sensitivity & $0.5 \mathrm{~K}$ \\
Antenna HPBW & $1^{\circ}$ \\
Scanning step & $0.4^{\circ}$ \\
Baseline length & $2 \mathrm{~m}$ \\
\hline
\end{tabular}

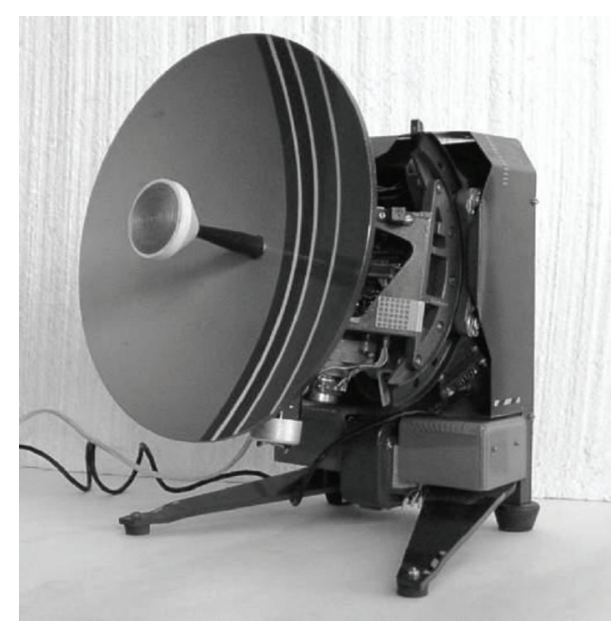

FIGURE 1: External appearance of the $3 \mathrm{~mm}$ imaging system for demonstration purposes.

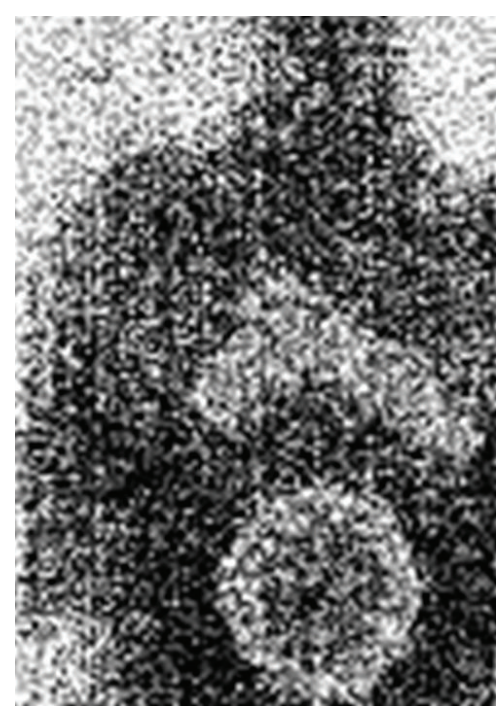

FIGURE 2: The radiometric image of the concealed objects on a person.

in a modular form, based on the single radiometric sensor shown in Figure 3.

The design and the realization of the array involved the following steps and requirements:

(a) good packing efficiency of the large number of receiving sensors in the array, by preserving high sensitivity of sensors; 


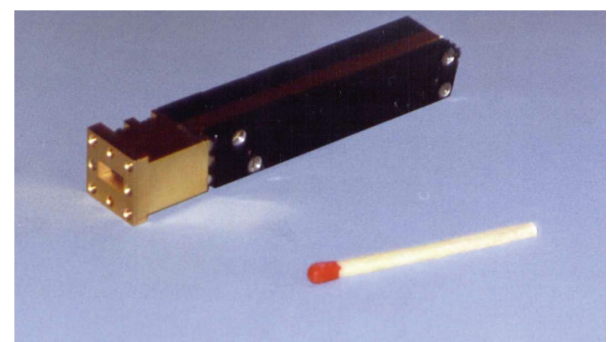

FIGURE 3: Single radiometric sensor.

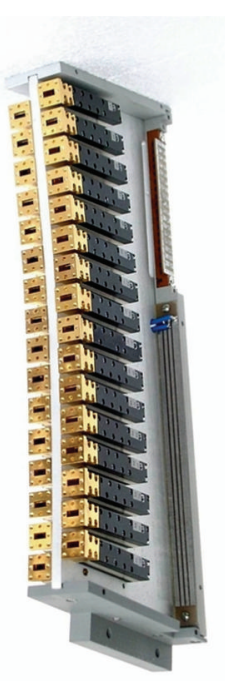

FIgURE 4: Array with 32 sensors.

(b) good characterization of the receiving channels in terms of electrical parameters;

(c) low mutual influence of receiving channels in the array;

(d) small weight, sizes, and cost of the array, of special concern for commercial applications;

(e) problem of power dissipation, which is worsened with the increase in the number of receiving channels.

Recent development in GaAs PHEMT technology makes it possible to realize the low-noise straight amplification receivers in the millimeter wave band, which are suitable for this radiometric array. The array contains 32 sensors aligned in the form of two vertical rows (Figure 4). Each row contains 16 sensors and the rows are shifted so as to form with antenna 32 beams, in this way, 32 strips will be achieved during the azimuth scanning of the array.

A large number of sensors composing the array arises the problem of power dissipation; in our case, each sensor gives off about $0.5 \mathrm{~W}$ in the form of heat, and hence the total power dissipation amounts to $16 \mathrm{~W}$. In order to dissipate the heat, all the sensors are fastened to the aluminum body with a radiator on the back side of the array.

A prime-focus-fed parabolic reflector is used as the main antenna. The reflector has diameter $D=900 \mathrm{~mm}$ providing a beam width near 0.6 degrees at $3 \mathrm{~dB}$ level. The focal length of the antenna is $F=990 \mathrm{~mm}$, which satisfies the condition $F / D \geq 1$ for multibeam antennas. Dielectric rod antennas $(\varepsilon=2,1)$ are used as feeds; this type of feed has smaller cross size compared to the horn feeds, allowing a more compact arrangement of the sensors in the array construction. Each feed is connected to the sensor and directed to the center of the main antenna. Feeds' axes form an angle of $48^{\circ}$ with the reflector axis, corresponding to a $7 \mathrm{~dB}$ gain in the radiation pattern of the reflector. The reflector with feeds in the focal plane forms 32 beams. The angle between adjacent beams in vertical plane is 0.48 degrees and total angle of view in vertical plane is 16 degrees.

Supply sources with stabilized voltages $+5 \mathrm{~V}$ and $\pm 12 \mathrm{~V}$ are common for all sensors. Output signals from the sensors are fed to an ADC card for digitization and subsequent processing, making use of a customized cable.

Sensitivity of the receivers in total power mode is better than $10 \mathrm{mK}$ with an integration time $\tau=1 \mathrm{~s}$. The microstrip construction of the receiving circuits has permitted to obtain small sizes of the sensor $(14 \times 13 \times 80 \mathrm{~mm})$ with a weight not exceeding 40 grams. The stretched configuration of the sensors (see Figure 4) helps to pack them efficiently into the receiving array. Waveguide input of each sensor is joined with the corresponding horn feed.

Antenna with array is moved by a scanning mechanism in the horizontal plane by an angle of \pm 60 degrees. At the endpoints of each scan, the calibration of the sensors is carried out. The calculation of the calibration factors is made possible by a digital processor.

During the scan, each beam draws the horizontal strip, and thus the observed scene find itself completely covered by beams. The scanning mechanism includes a rotary optical sensor, which gives information about the antenna position (angle) at any time during the system operation. The reading of the signals is carried out through each 0.17 degree (1 pixel) inside a field of view that spans $90^{\circ}$ in the horizontal plane.

The image of the survey scene is formed during each scan (3 seconds). In order to enhance quality of an image, the twoscan mode is provided by the system design. In this case, before the reverse scan, all beams are tilted by an angle of 0.24 degrees with the help of the reflector. As a result, during two scans the beams draw 64 strips, providing better resolution in the vertical plane.

The scanning mechanism is designed on the base of the usual asynchronous three-phase motor. The axis of the motor is mounted vertically. The reflector with the array is mounted on the motor's axis. The motor is powered by a converter, which transforms a single-phase voltage $(220 \mathrm{~V})$ into threephase pulse-duration voltage. The converter is controlled from the computer and provides switch-on and switch-off operation of the motor, the constant velocity of its rotation, and the smooth stop and reverse. In order to facilitate a load onto the motor at time of reverse, the special springs are used. Practically, the current is not going to the motor during the time of reverse. The external appearance of the passive imaging system is shown in Figure 5.

The list of the technical specifications is given in Table 2 . 
TABLE 2: The technical specifications of the $8 \mathrm{~mm}$ imaging system.

\begin{tabular}{lc}
\hline Operating wavelength & $8 \mathrm{~mm}$ \\
Temperature sensitivity & $\leq 10 \mathrm{mK} /(\mathrm{Hz})^{1 / 2}$ \\
Angular resolution & 0.3 degree \\
Image forming time & 3 seconds \\
Consumption power (mainly the driving motor) & $<0.3 \mathrm{~kW}$ \\
\hline
\end{tabular}

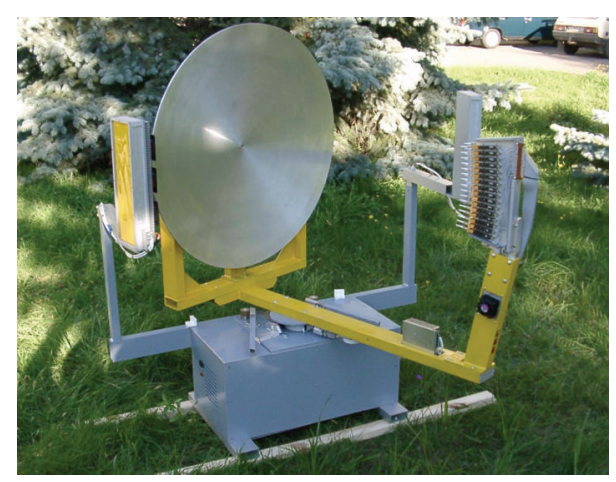

FIGURE 5: The $8 \mathrm{~mm}$ imaging system.

3.1. System Calibration and Control. The system is calibrated by following the steps below.

(i) Injection of the calibrating signals to the inputs of all the receiving channels.

(ii) Measurement of the levels of the output radiometric signals.

(iii) Evaluation of the correction factors for each channel.

The correction factors are then used for the processing of the measured radiometric signals, and a linear model is generally adopted for the conversion of the thermal radiation into the output signals of the radiometric receiver. Accordingly, the output signal of each channel can be represented in the form of the linear dependence as

$$
\begin{aligned}
C_{i} & =a_{i} T_{i}+b_{i}, \\
T_{i} & =\frac{C_{i}-b_{i}}{a_{i}},
\end{aligned}
$$

where $T_{i}$ is the effective temperature of the radiation from the target and $C_{i}$ is the output reading of $i$ th receiving channel; $a_{i}$ and $b_{i}$ are the factors expressing the linear dependence between the output receiving channel and the temperature. In order to build an image, an output binary code $C_{a}$ must correspond to the measured temperature $T_{i}$, with $C_{a}=A T_{i}$.

Therefore, the corrected output signal for the each channel $C_{a i}$ can be calculated by expression:

$$
C_{a i}=\frac{A\left(C_{i}-b_{i}\right)}{a_{i}} .
$$

Correction factors $a_{i}$ and $b_{i}$ can be determined during the active radiometric calibration of the channels by exploiting two reference radiators ("cold" and "hot"). These references have known temperatures of the radiation $T_{1}$ and $T_{2}$. In this case, the factors $a_{i}$ and $b_{i}$ can be determined according to the expressions

$$
\begin{gathered}
a_{i}=\frac{\left(C_{i 2}-C_{i 1}\right)}{\left(T_{2}-T_{1}\right)}, \\
b_{i}=\frac{\left(C_{i 1} T_{2}-C_{i 2} T_{1}\right)}{\left(T_{2}-T_{1}\right)},
\end{gathered}
$$

where $C_{i 1}$ and $C_{i 2}$ are the output readings of their channels corresponding to the two temperatures $T_{1}$ and $T_{2}$.

The hardware of the system includes also a PC and an $\mathrm{ADC} / \mathrm{DAC}$ card. The card is inserted into the computer and includes:

(i) 32 channels switch with analog-digital converter to read and digitize the signals from the sensors,

(ii) digital-analog converter for the computer control of scanning mechanism,

(iii) two digital inputs to transfer the information about the antenna position from the angle sensor to the computer.

Computer drives the operating modes for the scanning mechanism, carries out the processing of the received signals, and provides the image on the monitor screen. The software specifies the sequence of the system operations to provide fast and flexible control of the imaging system. The computer with the monitor is placed separately from the scanning receiver unit at a convenient distance and is connected to the unit by a proprietary customized cable, as mentioned before.

\section{The Image Processing}

Here, we present the results of simple denoising strategies applied to images achieved by the two systems. As can be seen, the two strategies are well tailored to the images acquired by the two systems, and the results are presented in two separate subsections for the two systems.

4.1. Denoising Strategies on Images with the $3 \mathrm{~mm}$ System. A denoising strategy has been applied to the images acquired by the $3 \mathrm{~mm}$ system, with the aim to reduce the fast variations occurring in the gray scale level of each image. The images are concerned with an urban scenario observed by the windows of a building (see Figures 6, 8, and 10).

The first data processing strategy consists in a simple multi-look (i.e., smoothing) procedure, which allows at mitigating this kind of disturbance; of course, this improvement is paid in terms of blurring effects on the images (see Figures 7,9 , and 11).

A more pleasant overall appearance can be obtained by employing a median filter instead, which exhibits good noise reduction capabilities, by arising less worsening in the shapes of the considered images (see Figures 7, 9, and 11). Median filtering is a nonlinear technique belonging to the family of the $i$ th order statistics filters. In particular, the $i$ th-order statistic filter replaces each pixel in the image to be processed 


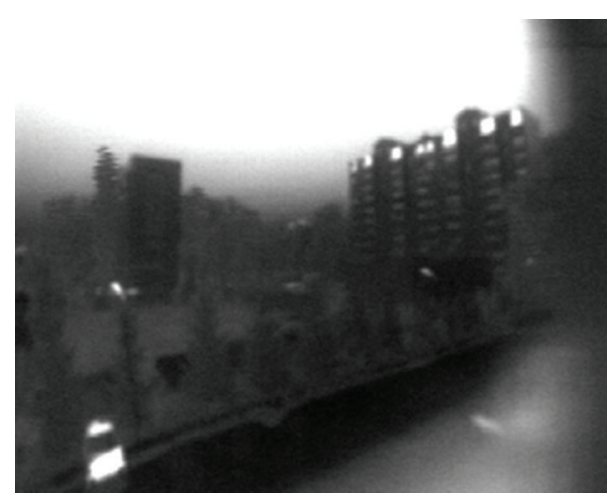

FIGURE 6: KUT25_WSS starting image.

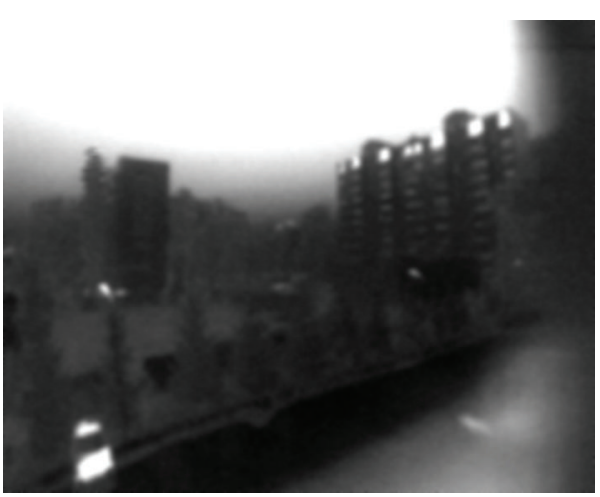

(a)

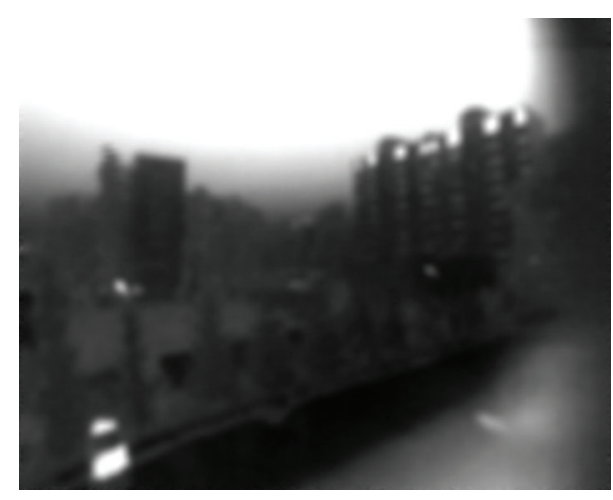

(c)

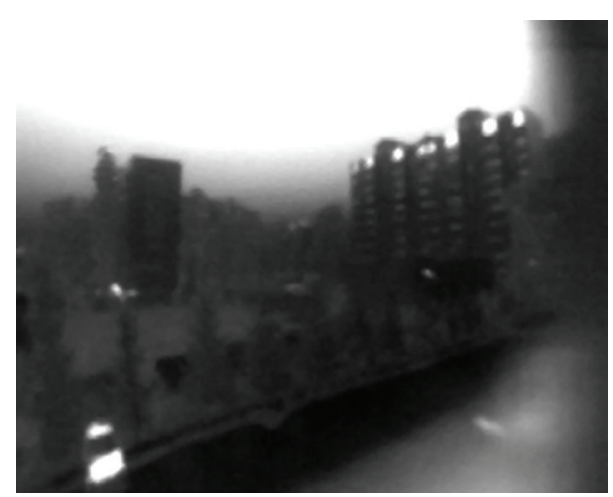

(b)

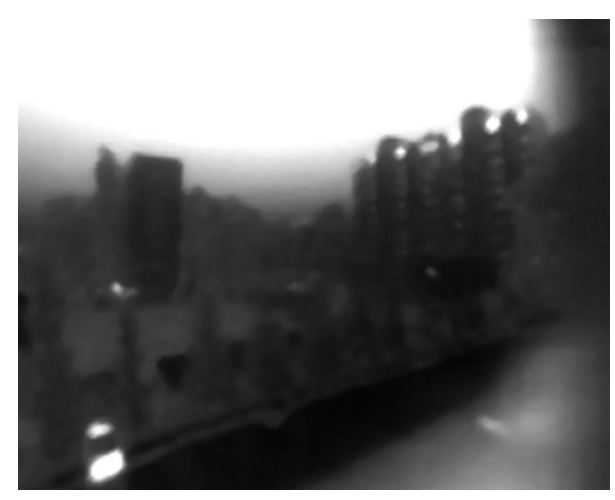

(d)

FIGURE 7: (a) Denoised KUT25_WSS $(3 \times 3$ averaging filter), (b) denoised KUT25_WSS $(3 \times 3$ median filter), (c) denoised KUT25_WSS $(5 \times$ 5 averaging filter), and (d) denoised KUT25_WSS $(5 \times 5$ median filter).

with the $i$ th percentile (i.e., the $i$ th-order statistic) of its neighbors, which is the $i$ th sample of the pixels belonging to the filter window, after they are ranked in ascending order of magnitude. As a special case, the choice $i=50$ defines the median filter, which is particularly effective at removing speckle and impulsive noise whilst preserving shapes and edges in the considered image $[9,10]$.

4.2. Denoising Strategies on Images with the $8 \mathrm{~mm}$ System. The dataset considered here is concerned with the possibility to detect objects in a long range; in particular, we are concerned with the detection of metallic panels of extent $1.25 \mathrm{~m} \times 2.5 \mathrm{~m}$ spaced each other by a $5 \mathrm{~m}$ distance, when the $8 \mathrm{~mm}$ imaging system was located at a distance of $500 \mathrm{~m}$ from the targets (see Figure 12). Figure 13 depicts the collected raw image and in this case, the image enhancement cannot be dealt with by the common denoising techniques, as in this case the main disturbance effect seems to be due to a (row-by-row) spacevariant gain of the acquisition instrument rather than the random noise.

Therefore, in order to compensate the gain variations, we subtract from each row its mean value; however, although 


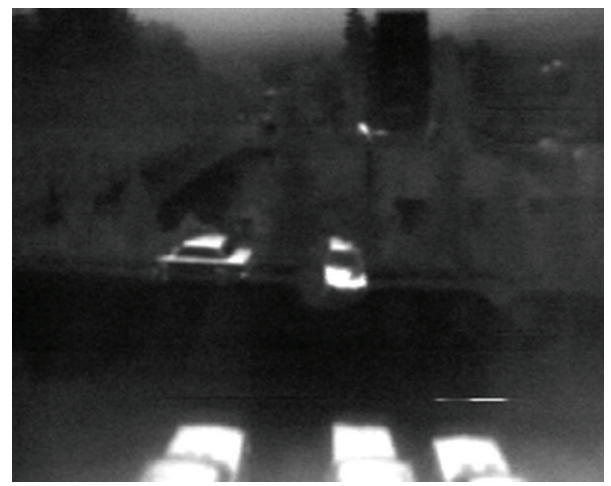

FIGURE 8: KUT26_WSS starting image.

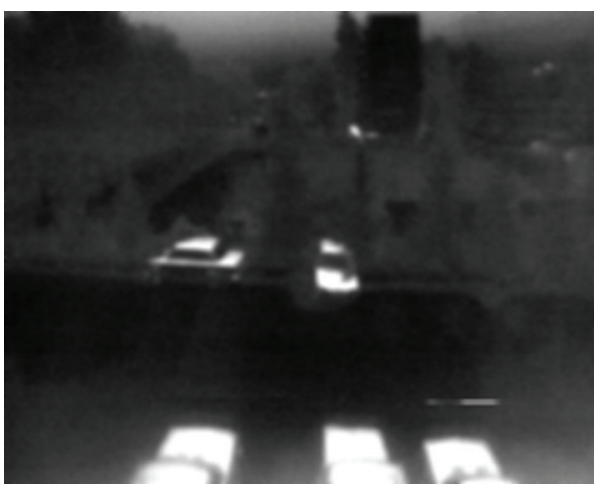

(a)

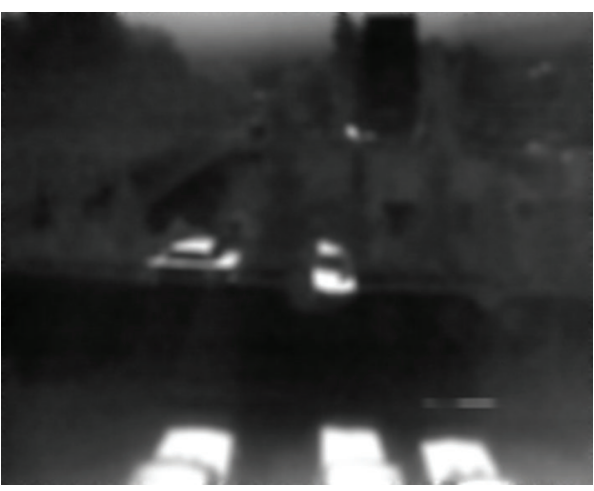

(c)

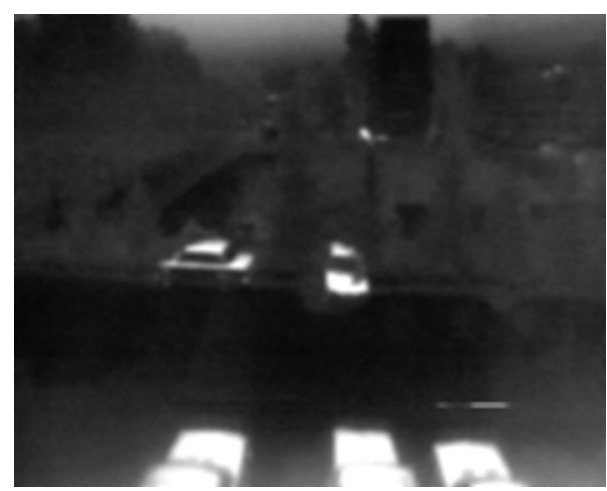

(b)

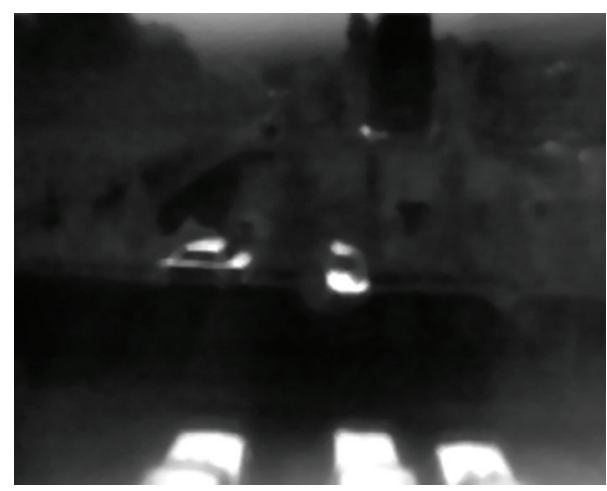

(d)

FIGURE 9: (a) Denoised KUT26_WSS ( $3 \times 3$ averaging filter), (b) denoised KUT26_WSS $(3 \times 3$ averaging filter), (c) denoised KUT26_WSS $(5$ $\times 5$ averaging filter), (d) and denoised KUT26_WSS $(5 \times 5$ median filter $)$.

such procedure allows reducing the unpleasant striping effect in the starting images (see Figure 14), it also causes the unnecessary beating down of the gray level in the bright zones (e.g., the sky regions), in which the gain fluctuations are less significant due to the saturation in the intensity of the pixels.

So, in order to overcome this drawback, we exclude the bright pixels from the evaluation of the local means; this strategy allows us to get high contrast images and preserve the desired enhancement (see Figure 15).

\section{Conclusion}

The paper has dealt with the description of two passive radiometric imaging systems developed at SRC for monitoring and surveillance issues. The design and the realization of the two systems have been performed by accounting for the requirements of a reasonable acquisition time and reliability and accuracy of the collected images. In this frame, the improvement of the interpretability of the images requires the 


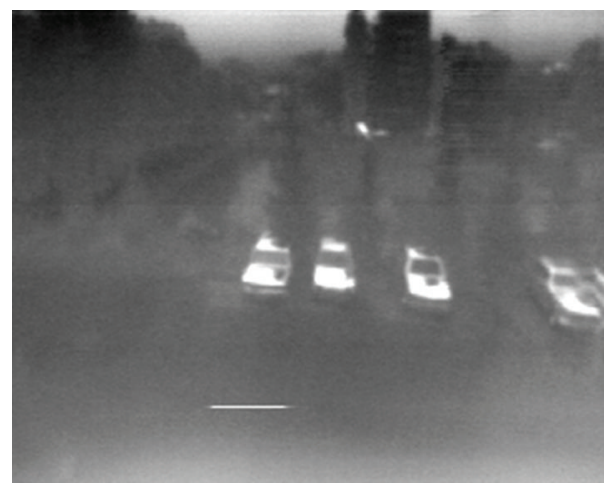

FIGURE 10: KUT28_WSS starting image.

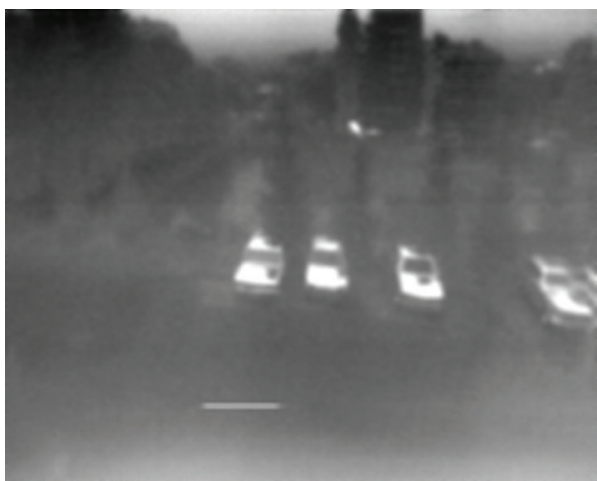

(a)

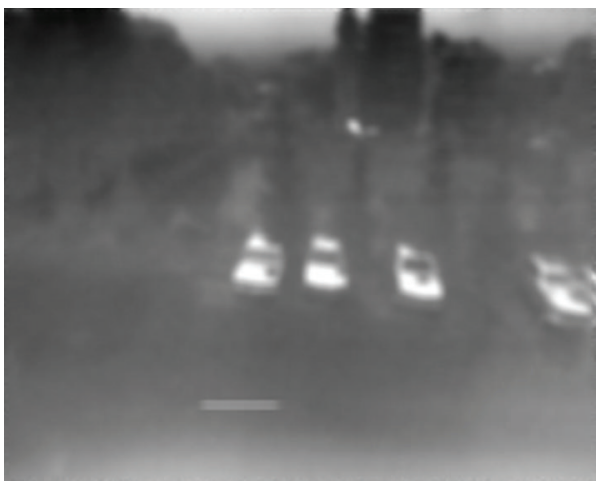

(c)

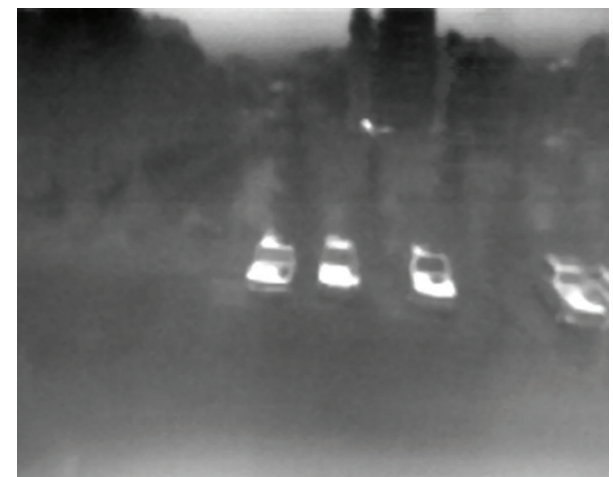

(b)

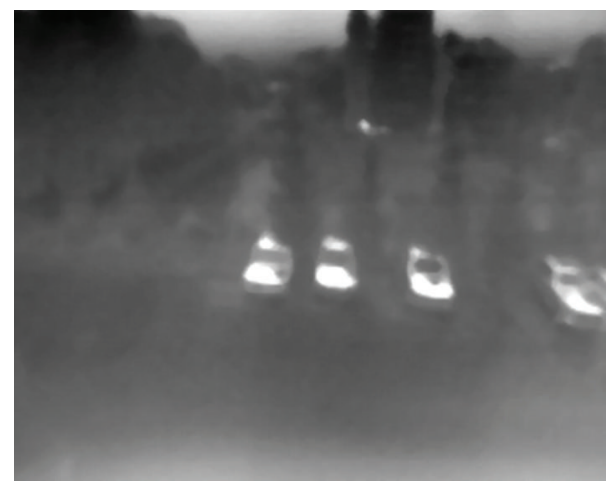

(d)

FIGURE 11: (a) Denoised KUT28_WSS ( $3 \times 3$ averaging filter), (b) denoised KUT26_WSS $(3 \times 3$ median filter), (c) denoised KUT28_WSS $(5 \times$ 5 averaging filter), (d) and denoised KUT28_WSS $(5 \times 5$ median filter $)$.

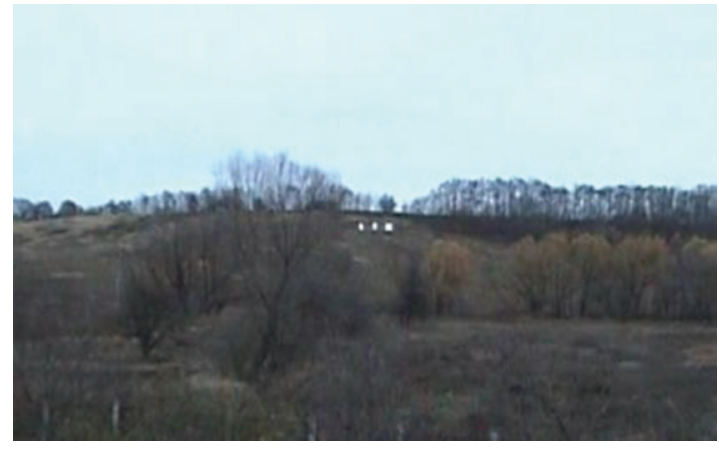

(a)

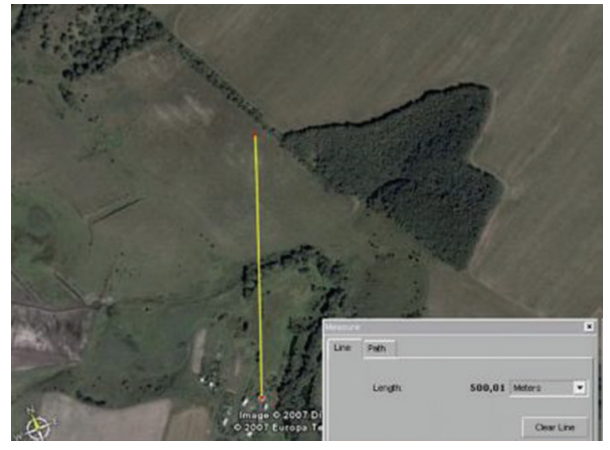

(b)

Figure 12: (a) Target scene; (b) Google image depicting the positions of the system and of the targets. 


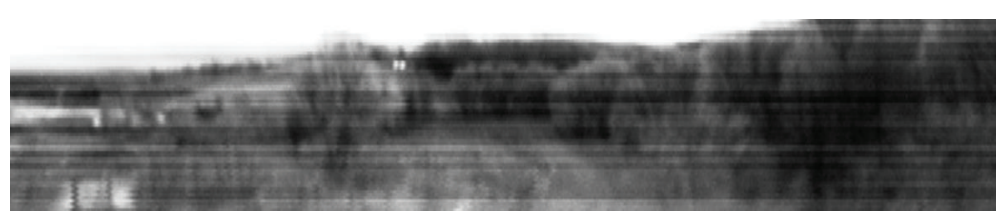

FIGURE 13: Starting image.

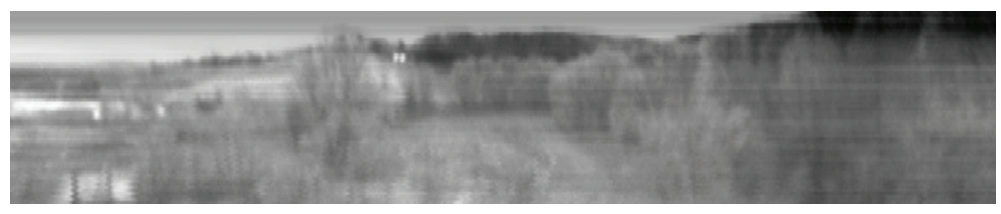

FIGURE 14: Image after the means subtraction.

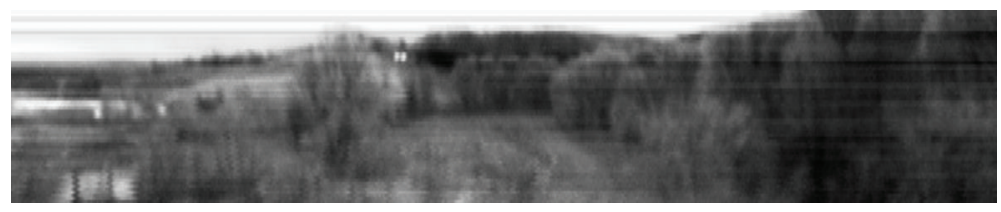

FigURE 15: Enhanced image where the means subtraction has been performed excluding the bright pixels.

development of ad hoc image filtering strategies, as shown in this paper.

\section{Acknowledgment}

This research has been performed in the framework of the "Active and Passive Microwaves for Security and Subsurface imaging (AMISS)" EU 7th Framework Marie Curie Actions IRSES project (PIRSES-GA-2010-269157).

\section{References}

[1] P. F. Goldsmith, C.-T. Hsieh, G. R. Huguenin, and J. Kapitzky, "Focal plane imaging systems for millimeter wavelengths," IEEE Transactions on Microwave Theory and Techniques, vol. 41, no. 10, pp. 1664-1675, 1993.

[2] A. Vertiy, S. Ozbek, A. Pavlyuchenko et al., "Short- and longrange passive imaging in millimeter-wave-band," in Proceedings of the 30th URSI General Assembly and Scientific Symposium (URSIGASS '11), pp. 1-4, August 2011.

[3] A. N. Radzikhovsky, V. P. Gorishniak, S. E. Kuzmin, and B. M. Shevchuk, "16-channels millimeter-waves radiometric imaging system," in Proceedings of the 4th International Kharkov Symposium on Physics and Engineering of Millimeter and Sub-Millimeter Waves (MSMW'01), pp. 466-468, Kiev, Ukraine, June 2001.

[4] V. N. Radzikhovsky, V. P. Gorishniak, S. E. Kuzmin, and B. M. Shevchuk, "Passive millimeter-wave imaging system," in Proceeding of the International Crimean Microwave Conference (CriMiCo '01), pp. 263-264, Crimea, Ukraine, September 2001.

[5] V. P. Gorishnyak, A. G. Denisov, S. E. Kuzmin, V. N. Radzikhovsky, and B. M. Shevchuk, "Radiometer imaging system for the concealed weapon detection," in Proceeding of the International Crimean Microwave Conference (CriMiCo '02), Crimea, Ukraine, September 2002.
[6] V. P. Gorishnyak, A. G. Denisov, S. E. Kuzmin, V. N. Radzikhovsky, and B. M. Shevchuk, " $8 \mathrm{~mm}$ passive imaging system with 32 sensors," in Proceedings of the 1st European Radar Conference (EuRAD '04), pp. 333-336, Amsterdam, The Netherland, October 2004.

[7] A. G. Denisov, V. P. Gorishnyak, S. E. Kuzmin et al., "Some experiments concerning resolution of 32 sensors passive $8 \mathrm{~mm}$ wave imaging system," in Proceedings of the International Symposium on Space Terahertz Technology (ISSTT '09), Charlottesville, Va, USA, April 2009.

[8] V. P. Gorishniak, A. G. Denisov, S. E. Kuzmin, V. N. Radzikhovsky, and B. M. Shevchuk, "Passive multichannels millimeterwaves imaging system," in Proceedings of the 5th International Kharkov Symposium on Physics and Engineering of Microwaves, Millimeter, and Submillimeter Waves (MSMW'04), pp. 202-204, June 2004.

[9] I. Pitas and A. N. Venetsanopoulos, "Order statistics in digital image processing," Proceedings of the IEEE, vol. 80, no. 12, pp. 1893-1921, 1992.

[10] G. R. Arce, Nonlinear Signal Processing: A Statistical Approach, John Wiley \& Sons, Hoboken, NJ, USA, 2005. 

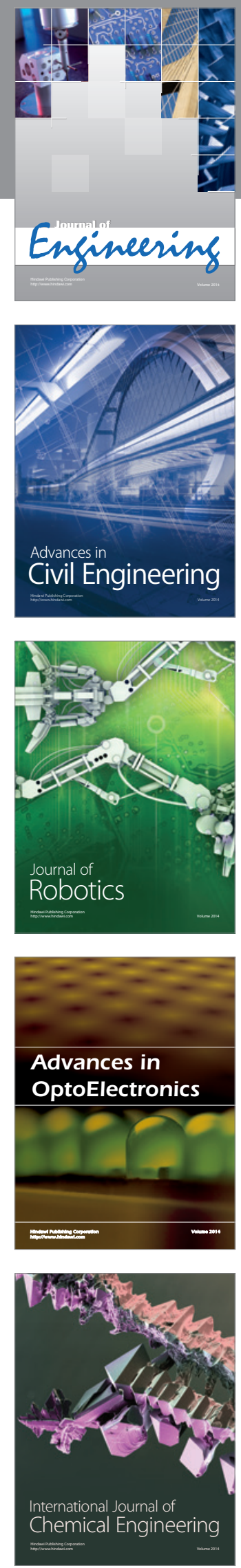

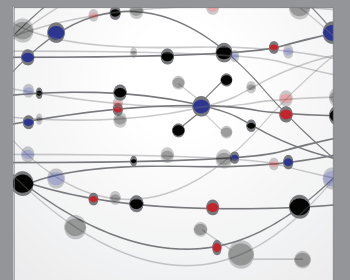

The Scientific World Journal
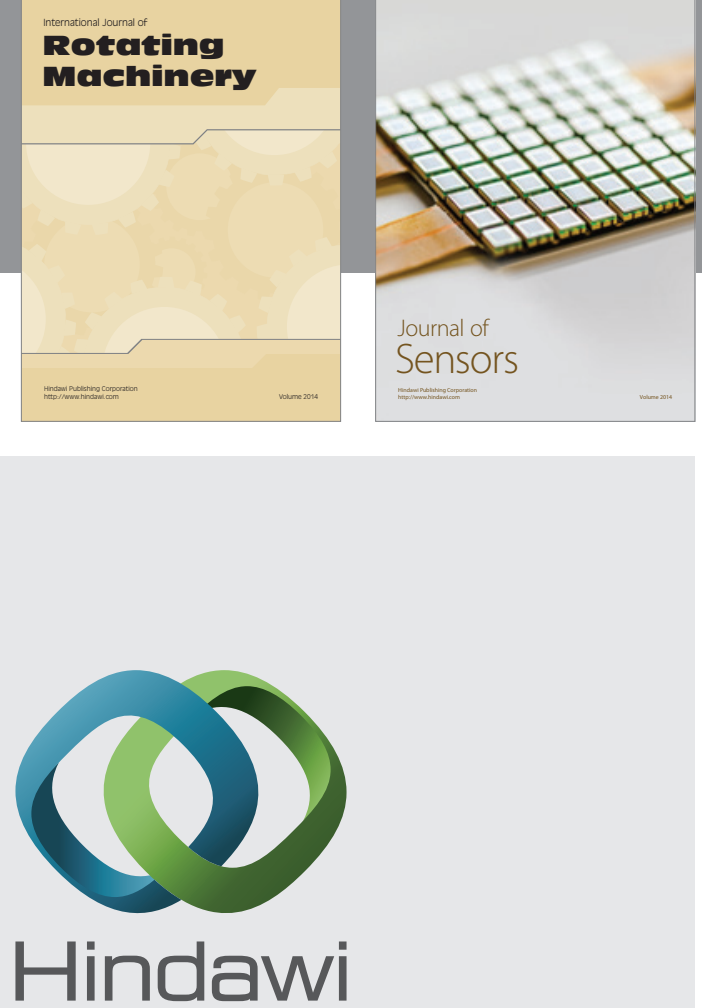

Submit your manuscripts at http://www.hindawi.com
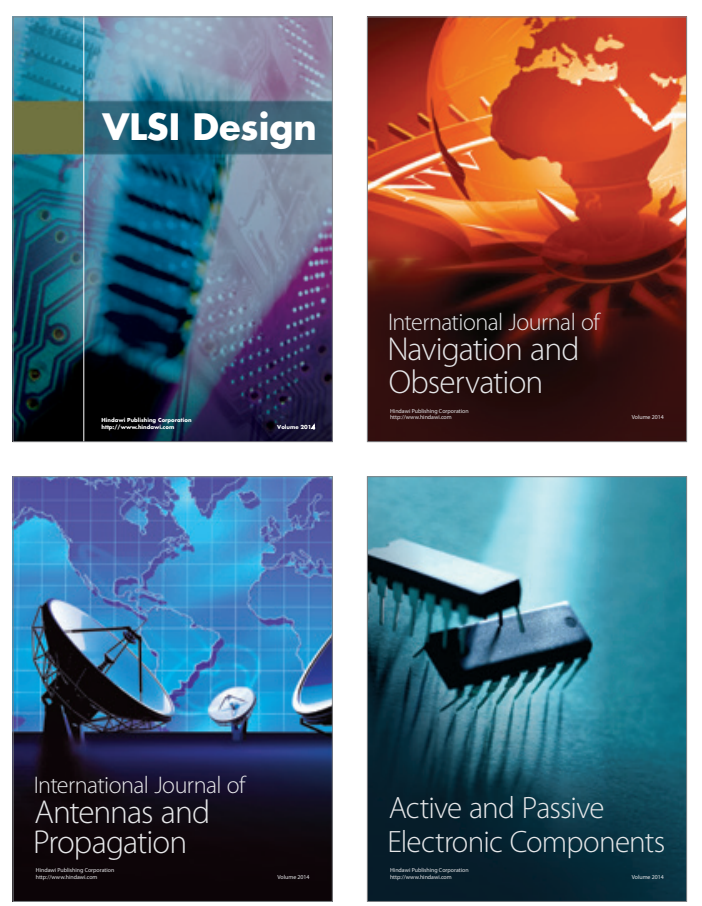
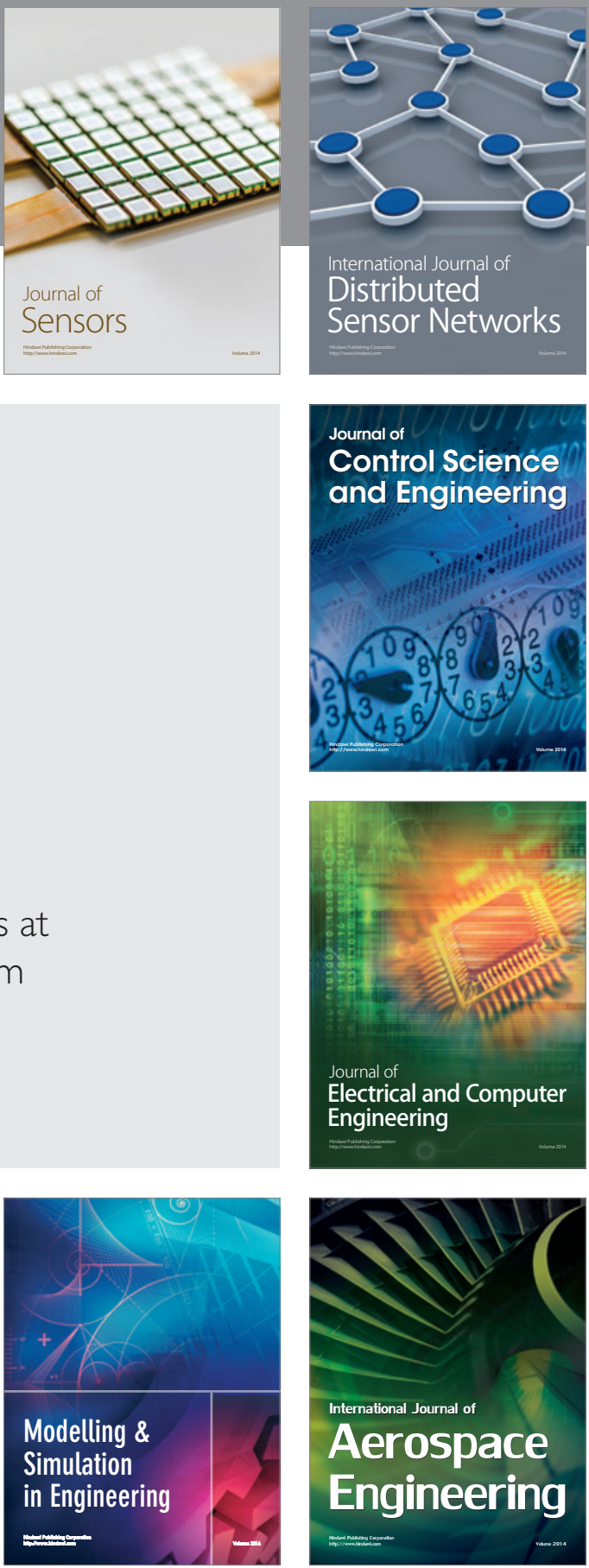

Journal of

Control Science

and Engineering
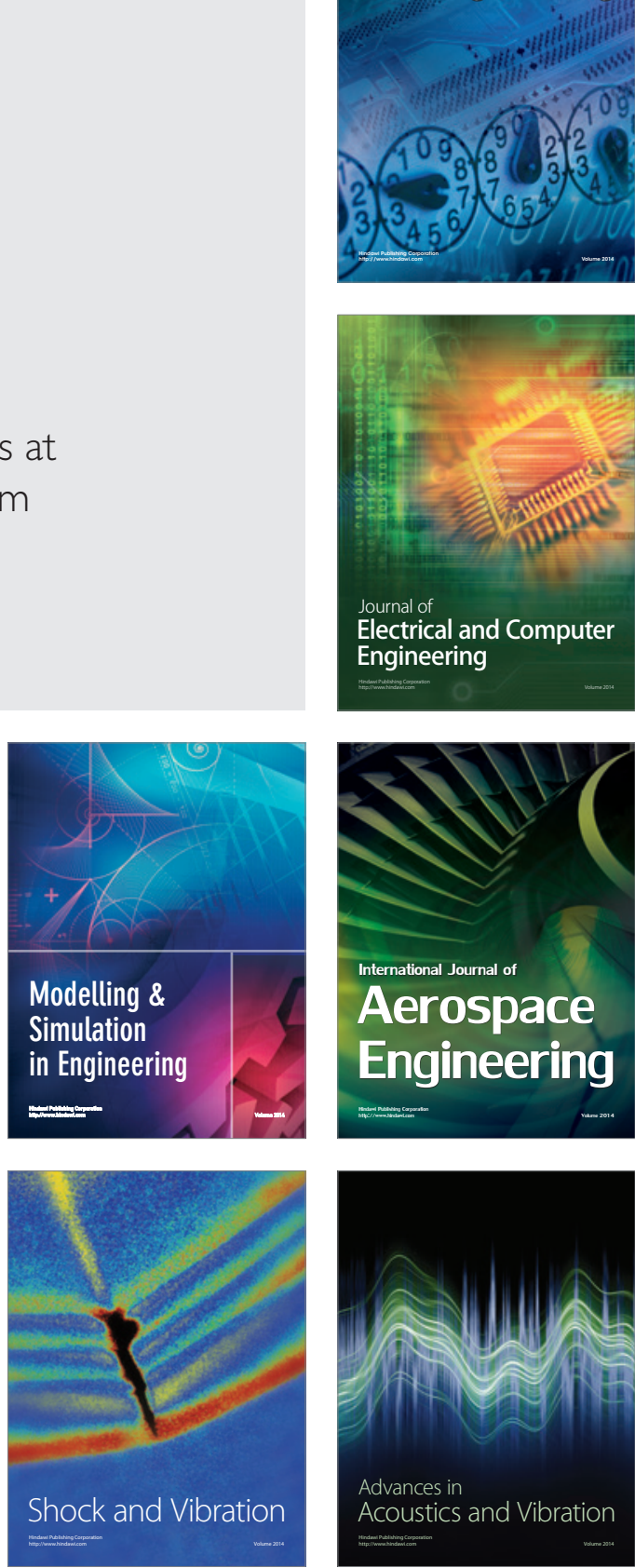